Guo, Qingbin

\section{Identification of c-myc target genes using a rat CDNA microarray}

\author{
Q ingbin Guo ${ }^{1}$, Mauro Ruffy ${ }^{1}$, R. Krishna Sanka ${ }^{1}$, Renae Malek², \\ Norman Lee $^{2} \&$ Edison T. Liu ${ }^{1}$
}

${ }^{1}$ Department of Cancer and Cell Biology, Division of Clinical Sciences, National Cancer Institute, Bethesda, M aryland 20892, USA

${ }^{2}$ The Institute for Genomic Research, Rockville, M aryland 20850, USA

The proto-oncogene $c-m y c$ encodes a highly conserved nuclear phosphoprotein that functions as a transcription factor in the cell. The discovery of deregulated $c-m y c$ in a variety of tumours and the ability of $c-m y c$ to transform cells either alone or in cooperation with the ras oncogene indicate that the activation of $c$ $m y c$ is involved in tumorigenesis. In addition to its tumorigenic ability, $c-m y c$ has also been implicated in apoptosis and the control of normal cell growth. The identification and characterization of the target genes of $c-m y c$ may help us to decipher the mechanism by which $c$-myc functions in various biological processes. To identify the genes regulated by $c-m y c$, we compared the gene expression profiles between $c-m y c$ wild-type and $c$-myc mutant rat fibroblast cells using the cDNA glass microarray method. After rat EST clones were sequence verified and annotated, we assembled unique 4,400-element cDNA microarrays on glass slides. Using these microarrays, we studied differential gene expressions between wild-type $c-m y c$ and $c-m y c^{-/}$rat fibroblast cells under different conditions. We confirmed the differential expression of some candidate genes by northern blot. We hope that these microarray studies may elucidate the pathways that are regulated by $c-m y c$, which may in turn help us to understand the mechanism by which $c-m y c$ functions in normal, neoplastic or apoptotic cells.

\section{Hardwick, James}

\section{The transcriptional profile of Saccharomyces cerevisiae exposed to rapamycin mimics the profile induced by amino acid starvation}

\section{James S. Hardwick, Jeffrey K. Tong \& Stuart L. Schreiber}

Howard Hughes M edical Institute, Harvard Center for Genomics Research, Department of Chemistry \& Chemical Biology, Harvard University, 120 xford Street, Cambridge, M assachusetts 02138, USA

Rapamycin is a potent immunosuppressant that affects the cellular protein translation machinery. The cellular targets of rapamycin, FRAP/RAFT1/mTor in mammals and Tor1p/Tor $2 p$ in Saccharomyces cerevisiae, are members of the PI3kinase related family of protein kinases. Rapamycin inhibits the catalytic activity of these serine protein kinases while at the same time activating protein phosphatase $2 \mathrm{~A}$, events that have the effect of sharply reducing the phosphorylation and function of $\mathrm{p} 70^{S \sigma K}$ and eIF-4E binding protein. Recent studies have suggested that FRAP is involved in a cell-cycle checkpoint mechanism that senses intracellular nutrient availability by sampling levels of free amino acids. Here we used DNA microarrays to investigate the effects of rapamycin exposure or amino acid deprivation on the transcriptional program of the budding yeast $S$. cerevisiae. We show that over a period of two hours the transcriptional response to rapamycin exposure is similar to the response following amino acid starvation in both haploid and diploid cells. The transcriptional response to rapamycin appears to be a subset of a more extensive transcriptional response induced by amino acid withdrawal. Our results suggest that Tor protein inactivation elicits most features of a nitrogen starvation response in yeast even in the presence of abundant nutrients. Our results also indicate the ease with which a genome-based approach is able to identify the intracellular pathway targeted by a small molecule drug.

Hawthorn, Lesleyann

\section{Increased levels of human telomerase activity have been demonstrated in a wide variety of human tumours}

\author{
Lesleyann Hawthorn
}

Cleveland Clinic Foundation, Cleveland, O hio, USA

We have used a 19mer, antisense oligonucleotide directed against the human telomerase RNA component (hTR) to inactivate this enzyme. These oligos are linked to a $2^{\prime}, 5^{\prime}$-oligoadenylate moiety which activates RNAase $\mathrm{L}$, resulting in degradation of the target RNA. Targeting hTR in variety of human tumour cells from different origins in vitro results in drastically reduced cell viability. In vivo tumours grown subcutaneously in nude mice reduce in size following treatment with the anti-hTR oligos. Normal fibroblast and epithelial cells do not show any reduction in viability following treatment. TUNEL assays demonstrate that the cytotoxic effects are mediated through apoptotic pathways where the maximum effect is seen after only 4 days of treatment. This response is p53 independent since the majority of the tumour cells used are deficient in p 53 function. It also seems that telomere shortening is not the cause of cell death since the cells do not go through sufficient rounds of division for this to happen. In order to gain more insight into the pathways which are affected by the 2-5A anti-hTR treatment we investigated the changes in gene expression which are produced in the human glioma cell line U373 following 2-5A anti-hTR treatment using Affymetrix GeneChip microarray technology. We compared gene expression levels at 4 different time points after treatment using the Affymetrix HU6800 chips. The data demonstrates dysregulation of a significant number of genes implicating the possible involvement of a number of different pathways. The role of these genes in the response to inactivation of telomerase is currently under investigation.

\section{Hayashibara, Kathleen}

\section{Mapping single and multigenetic traits in S. cerevisiae by genomic mismatch scan- ning and DNA microarrays}

\author{
Kathleen C. Hayashibara ${ }^{1}$, Esha Ray ${ }^{1}$, David Elashoff', Joseph \\ DeRisi ${ }^{1}$, John H. McCusker ${ }^{3}$, David 0. Siegmund ${ }^{2}$ \\ \& Patrick 0. Brown ${ }^{1}$
}

${ }^{1}$ Howard Hughes M edical Institute/ Stanford University Department of Biochemistry, Stanford, California 94305, USA

${ }^{2}$ Department of Statistics, Stanford University, Stanford, California 94305, USA

${ }^{3}$ Duke University Department of M icrobiology, Durham, North Carolina 27710, USA

The increasing abundance of sequence data has facilitated the task of mapping genetic traits in several organisms by expanding the number of highly informative locusspecific markers for linkage analysis. However, the need exists for efficient, costeffective methods for high-resolution genetic mapping. Genomic mismatch scanning (GMS) is a technique that utilises mismatch repair enzymes from Escherichia coli to screen for polymorphisms between genomic DNA samples from related individuals to select for regions of identity by descent (IBD) simultaneously. Regions of IBD selected by GMS are identified by hybridisation to a microarray of mapped DNA elements. No prior knowledge of the location of sequence polymorphisms within the 